\title{
Síndrome de Hiper-IgM en miembros de 2 familias chilenas no relacionadas: Análisis genético- molecular
}

\author{
Pamela Luttges $D^{1 \mathbf{a}}$, David Retamal $F^{1 a}$, Michael Spencer $Y^{1}$, \\ Flavio Carrión $A^{2 b}$, Viviana Valenzuela $\mathbf{M}^{2 a}$, Sara Navarro $V^{1 c}$, \\ Mónica Cornejo De L'ำ. \\ Hyper-IgM Syndrome in members of \\ two unrelated Chilean families: \\ molecular and mutation analysis
}

\begin{abstract}
Background: Hyper-IgM syndrome (HIGM) is a rare primary immunodeficiency used to describe a heterogeneous group of disorders characterized by recurrent bacterial infections, normal or elevated serum IgM levels and low or absent serum IgG, IgA and IgE. Aim: To make definitive diagnosis, detect mutations in carriers and perform genetic counseling in patients with HIGM. Patients and methods: We studied the expression of CD40L, CD40 and made a mutation analysis of the CD40L gene in 3 males of 2 unrelated Chilean families diagnosed as a possible syndrome of hyper-IgM and 3 relatives. Results: We identified a deletion frameshift in the exon 2 (delA225) of the extracellular domain of CD40L gene in one patient and verified the carrier status of his mother and sister. The other patients showed a low expression of CD40L in activated T cells (65.3\% and 65.5\%) and a normal expression of CD40. No alterations were found in the single strand conformation polymorphism analysis of the CD40L Conclusions: These result allowed us to make a definitive diagnosis of HIGM1 of a patient, detect female carriers and suggest a HIGM of recessive inheritance with normal CD40 expression in the patients of the second family (Rev Méd Chile 2004; 132: 1179-88).
\end{abstract}

(Key Words: CD40-binding protein, human; Immunoglobulin M; Mutation)

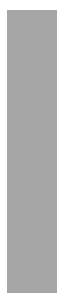

\footnotetext{
Recibido el 15 de enero, 2004. Aceptado en versión corregida el 19 de agosto, 2004.

Trabajo financiado por la Dirección de Investigación y Posgrado de la Universidad de Valparaíso. Proyectos Dipuv 11/99 y 05/02.

${ }^{1}$ Laboratorio de Inmunología, Facultad de Medicina, Universidad de Valparaíso. ${ }^{2}$ Laboratorio de Inmunología, Facultad de Medicina, Universidad de Los Andes.

aBioquímico

bBioquímico, $\mathrm{PhD}$

cBióloga
}

Correspondencia a: Pamela Luttges D. Laboratorio de Inmunología, Facultad de Medicina, Universidad de Valparaíso. Hontaneda 2653, Valparaíso, Chile. Fono: 5632-507369. Fax: 56-32-507321. E mail: pamela.luttges@uv.cl 
$\mathrm{E}^{1}$ Síndrome de Hiper-IgM (HIGM) es una sinmunodeficiencia primaria poco frecuente, caracterizada por infecciones recurrentes y niveles plasmáticos normales o elevados de $\operatorname{IgM}$ y niveles disminuidos o ausentes de IgG, IgA e IgE. En 1993, varios grupos demostraron que la causa etiológica de este síndrome son mutaciones en el gen que codifica el ligando de CD40 (CD40L o CD154 o TNFSF5). Esta molécula se expresa en la superficie de linfocitos T (LT) CD4+ activados y en menor proporción en los LT CD8+, mastocitos, basófilos y plaquetas. Se une a la molécula CD40 (Figura 1) expresada constitutivamente en la superficie de los linfocitos B (LB), macrófagos, células dendríticas y células epiteliales, generando una cascada de eventos intracelulares que activan el factor nuclear $\mathrm{kB}(\mathrm{NFkB})$, que en el caso del LB son fundamentales para su proliferación, cambio de isotipo de cadena pesada de inmunoglobulinas y formación de centros germinales en el linfonodo. Recientemente, se ha descrito que defectos en el CD40 o en otras moléculas relacionadas con la vía de activación del LB también producen el mismo síndrome.
Al HIGM por déficit de CD40L se le denomina HIGM1, se hereda típicamente como un rasgo genético ligado al cromosoma $\mathrm{X}$ y representa el $65 \%$ de los pacientes con HIGM ${ }^{1}$. El 15\% presenta una forma autosómica recesiva del síndrome relacionada con mutaciones en el gen que codifica para una citidín deaminasa (activating induced cytidine deaminase, AID) ${ }^{2}$ parte de la cascada de activación del LB e implicada en el cambio de isotipo, hipermutación somática y formación de centros germinales. Menos de 5\% presenta mutaciones en el gen que codifica para la molécula CD403 imprescindible en el desarrollo, crecimiento y diferenciación de los LB. Estos síndromes se denominan actualmente HIGM2 y HIGM3, respectivamente. Se han descrito otras dos formas recesivas $^{4,5}$ de la enfermedad. La primera, denominada HIGM4, cuyos mecanismos moleculares son aún desconocidos y la segunda, llamada HIGM5, producida por mutaciones en el gen de una glicosilasa (uracil DNA glycosylase, UNG) que participa junto con AID en el cambio de isotipo e hipermutación somática de anticuerpos. Por otro lado, el 10\% de los pacientes con HIGM presenta

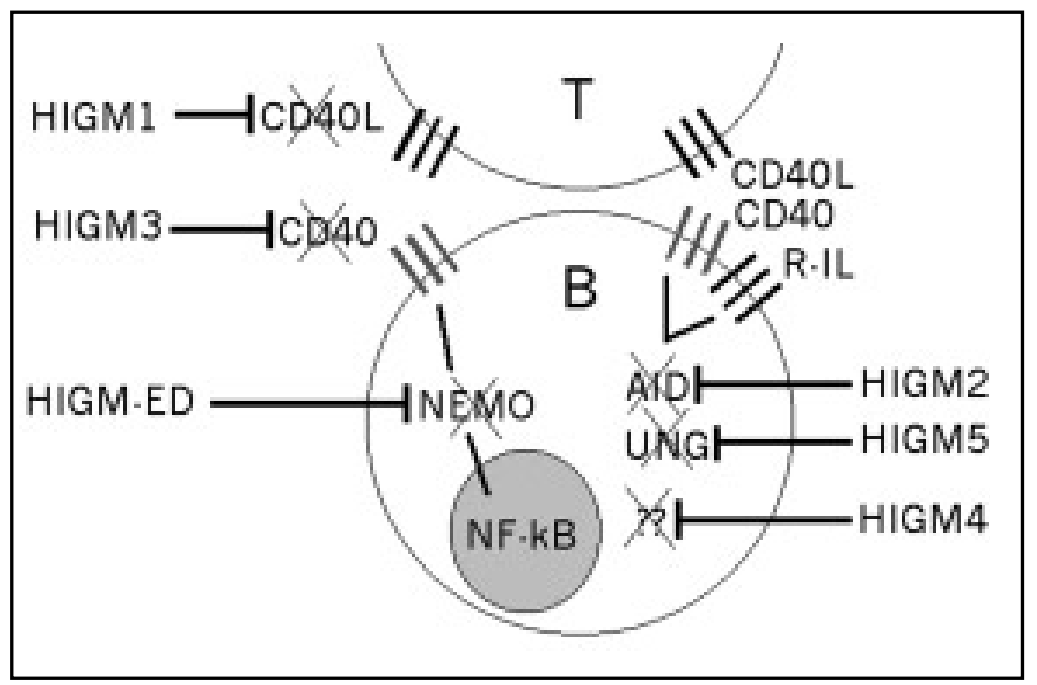

FIGURA 1. Representación esquemática de las bases moleculares del síndrome de hiper-IgM. La interacción entre el CD40L presente en el LT activado y el CD40 en presencia de citoquinas, es esencial para la proliferación del LB, cambio de isotipo de cadena pesada de inmunoglobulinas, maduración de afinidad de la inmunoglobulina y formación de centros germinales en el linfonodo. Defecto en los receptores de membrana (CD40L y CD40) 0 en la cascada de activación del NF-kB (NEMO) o en las enzimas AID y UNG que se producen por la unión de los receptores CD40L-CD40 en presencia de citoquinas, afectan en forma similar la diferenciación terminal del LB y producen el síndrome de hiper-IgM. 
mutaciones en el modulador esencial de la molécula $\mathrm{NFKB}$ conocida como $\mathrm{NEMO}^{1}$, que produce una displasia ectodérmica hipodrótica asociada a HIGM $^{6}$. Todos estos síndromes presentan características clínicas muy similares y sólo a través de estudios moleculares y genéticos se puede realizar un diagnóstico diferencial ${ }^{7}$.

El CD40L es una glicoproteína que actúa como factor de crecimiento, inducción y activación de los LT. Se expresa en membrana como homotrímero y está compuesta por 261 aminoácidos. Consta de una región citoplasmática corta, una transmembrana y una gran porción extracelular. Pertenece a la familia de los receptores del TNF y se codifica en el cromosoma Xq26.3 en 5 exones. Se han descrito mutaciones en toda la molécula CD40L sin correlación genotipo-fenotipo aparente ${ }^{8}$. En la porción más extracelular se encuentra la zona con homología TNF que interacciona con el receptor. Esta zona es codificada por parte del exón 4 y todo el exón 5 , y concentra la proporción más alta de mutaciones (65\%).

El objetivo de este trabajo fue analizar, a nivel molecular y genético, las moléculas CD40 y CD40L en 3 pacientes chilenos con diagnóstico posible de HIGM, con el fin de confirmar el diagnóstico. También se estudian familiares directos para detectar eventuales portadores y poder entregar consejo genético.

\section{PACIENTES Y MÉTODOS}

Pacientes y controles. Se estudiaron 3 varones pertenecientes a 2 familias no relacionadas de la $\mathrm{V}$ región, Chile. Los pacientes 1 y 2 (P1 y P2) de la familia 1, provenían de un matrimonio consanguíneo (primos). En la familia del paciente 3 (P3) existían antecedentes de varones (hermano y tío materno) fallecidos a temprana edad. Los pacientes fueron clasificados como HIGM posible, de acuerdo a los criterios diagnósticos para Inmunodeficiencias Primarias (IDP), publicados en 1999 por el Grupo Panamericano de IDP (PAGID) y la Sociedad Europea de IDP (ESID) ${ }^{9}$. Las características clínicas e inmunológicas de los pacientes se muestran en la Tabla 1. Desde el momento del diagnóstico, todos recibieron terapia de reemplazo con gammaglobulina. Se incluyeron en este estudio, 3 familiares directos, de sexo femenino, sin antecedentes clínicos ni de laboratorio de HIGM. Los controles fueron individuos sanos, de sexo y edad similar, no relacionados con los pacientes, sin antecedentes personales o familiares de HIGM y provenientes de la misma área geográfica. Todos los pacientes, familiares y controles firmaron un consentimiento informado aprobado por el Comité de Ética de la Escuela de Medicina de la Universidad de Valparaíso.

Cuantificación de la expresión de CD40 y CD40L La expresión de CD40 y CD40L en linfocitos de sangre total fue determinada mediante citometría de acuerdo a lo descrito por $\mathrm{O}^{\prime} G o r m a{ }^{10}$ con ligeras modificaciones. Para la medición de CD40L, sangre total heparinizada fue estimulada durante $6 \mathrm{~h}$ en ausencia 0 presencia de forbol miristato acetato (PMA) e ionomicina a $37^{\circ} \mathrm{C}$ y $5 \%$ de $\mathrm{CO}_{2}$. Posteriormente, la sangre fue teñida con anticuerpos monoclonales utilizando un panel de 3 colores (CD3-FITC/CD40L-PE/CD8-TC). Como control positivo de la estimulación se utilizó la

Tabla 1. C aracterísticas clínicas e inmunológicas de los pacientes

\begin{tabular}{|lcccccccccccccc|}
\hline $\begin{array}{c}\text { Familia } \\
\text { y } \\
\text { paciente }\end{array}$ & $\begin{array}{c}\text { Edad } \\
\text { al } \\
\text { diagnóstico }\end{array}$ & IR & I0 & Neutropenia & IB & & & & & & & & &
\end{tabular}

IR: Infecciones recurrentes; IO: Infecciones oportunistas; -: negativa; t: escasa; +: recurrentes; +t: severas; nd: no detectable; N: normal; $\uparrow$ sobre 2 DS para la edad; $\downarrow$ bajo 2DS para la edad. 
expresión del marcador temprano de activación CD69. La medición de la expresión de CD40, se realizó en sangre no estimulada utilizando un panel de 2 colores (CD19-FITC/CD40-PE). Un mínimo de 10.000 células por muestra fueron adquiridas para el análisis. La adquisición y el análisis fue realizado en un citómetro Coulter Epics-XL.

Aislamiento de ADN genómico y RPC para los 5 exones de la molécula CD40L A partir de $5 \mathrm{ml}$ de sangre total con anticoagulante EDTA, se obtuvo el ADN genómico de pacientes, familiares y controles por la técnica del DNAzol (Invitrogen). La reacción de Polimerasa en Cadena (RPC) se realizó en un termociclador marca Thermo Hybaid modelo PCR Sprint. El volumen final de la reacción fue de $50 \mu \mathrm{l}$ conteniendo $200 \mathrm{ng}$ de $\mathrm{ADN}, 10$ $\mathrm{mM}$ Tris- $\mathrm{HCl} \mathrm{pH}=8,3,1,5 \mathrm{mM} \mathrm{MgCl} 2,0,5 \mu \mathrm{M}$ de cada partidor, 2,5 $\mathrm{U}$ de Taq polimerasa (Invitrogen) y $200 \mu \mathrm{M}$ de cada dNTP. La secuencia de los partidores utilizados se muestra en la Tabla 2. Las condiciones de reacción fueron: 2 min a $95^{\circ} \mathrm{C}, 35$ ciclos de $5 \mathrm{~s}$ a $94^{\circ} \mathrm{C}, 15 \mathrm{~s}$ a temperatura de apareamiento respectiva, $30 \mathrm{~s}$ a $72^{\circ} \mathrm{C}$, y una extensión final de $7 \mathrm{~min}$ a $72^{\circ} \mathrm{C}$. La pureza de los productos amplificados se determinaron por electroforesis en agarosa al 1,5\% teñida con bromuro de etidio.

Análisis de Polimorfismos Conformacionales de Cadena Simple (SSCP). Volúmenes iguales de los productos amplificados con tampón de carga $(0,05 \%$ de azul de bromofenol, 0,05\% cyanol xylene, 95\% formamida y $20 \mathrm{mM}$ EDTA) fueron denaturados a $90^{\circ} \mathrm{C}$ por $10 \mathrm{~min}$ y colocados inmediatamente en hielo. Se realizó una electroforesis no denaturante en un gel de poliacrilamida al $6 \%$, TBE $0,5 \mathrm{X}$, glicerol al $8 \%$ a $2 \mathrm{~W}$ toda la noche a temperatura ambiente. Las bandas de ADN fueron teñidas con plata.

Secuenciamiento del ADN. Los productos de RPC que dieron bandas alteradas en el SSCP se purificaron con el kit Concert ${ }^{\mathrm{TM}}$ Rapid Purification System (Life Technologies) de acuerdo a las especificaciones del fabricante. Los productos purificados fueron secuenciados con técnicas estándares en un secuenciador automático $\mathrm{ABI}$ Prism 310 Applied Biosystems.

\section{Resultados}

Cuantificación de la expresión de CD40L y CD40 en pacientes con HIGM y controles. Los estudios de expresión de CD40 y CD40L se realizaron en los pacientes P1 y P2, y no fue posible realizarlo en el paciente P3. Los pacientes P1 y P2 tuvieron una expresión basal de CD40L de 0,2\% en ambos casos, mientras tras estimulación con PMA e ionomicina la expresión de CD40L fue de 65,3\% y $65,5 \%$, respectivamente (Figura 2). En el control, la expresión basal de CD40L fue de 0,3\% y tras estimulación con PMA e ionomicina fue de 92,3\% (Figura 1). La expresión de CD69, utilizada como control de la técnica, fue similar tanto en los pacientes P1 y P2 como en el control tras estimulación con PMA e ionomicina (99,6\%, 99,9\% y $99,9 \%$, respectivamente). El porcentaje de expresión de CD40 en los linfocitos B de los pacientes P1, P2 y el control fue de $94,5 \%, 96,4 \%$ y $99,4 \%$.

Tabla 2. Secuencia de partidores utilizados para el estudio de mutaciones de la molécula CD 40L

\begin{tabular}{|lllccc|}
\hline Par & Forward & Reverse & Exón & Largo $(\mathrm{pb})$ & $\mathrm{T}^{\circ}$ \\
\hline 1 & TTCTCTGCCAGAAGATACC & CATTTGGGTAGAACCAACC & 1 & 249 & $50^{\circ}$ \\
2 & GCTGTATTCTCCTTCCGAA & TGGCATTGTCAGTTCC & 2 & 246 & $50^{\circ}$ \\
3 & AGCAGAGCCCCTGTCAAAATGAC & CCAAATCACAGGGACCAGAGAAGG & 3 & 250 & $60^{\circ}$ \\
4 & CCTTCAGTGGGAGAGATGTCAGAC & ATGACTTCGGCATCCCAGCCTT & 4 & 256 & $60^{\circ}$ \\
5 & GCTTCACCTCACCACAAACTTTCC & CGGAACTGTGGGTATTTGCAGCT & $5 \mathrm{a}$ & 250 & $60^{\circ}$ \\
6 & CCATTTATAGCCAGCCTCTGCCTA & TATGAAGACTCCCAGCGTCAGCT & $5 \mathrm{~b}$ & 252 & $60^{\circ}$ \\
\hline
\end{tabular}




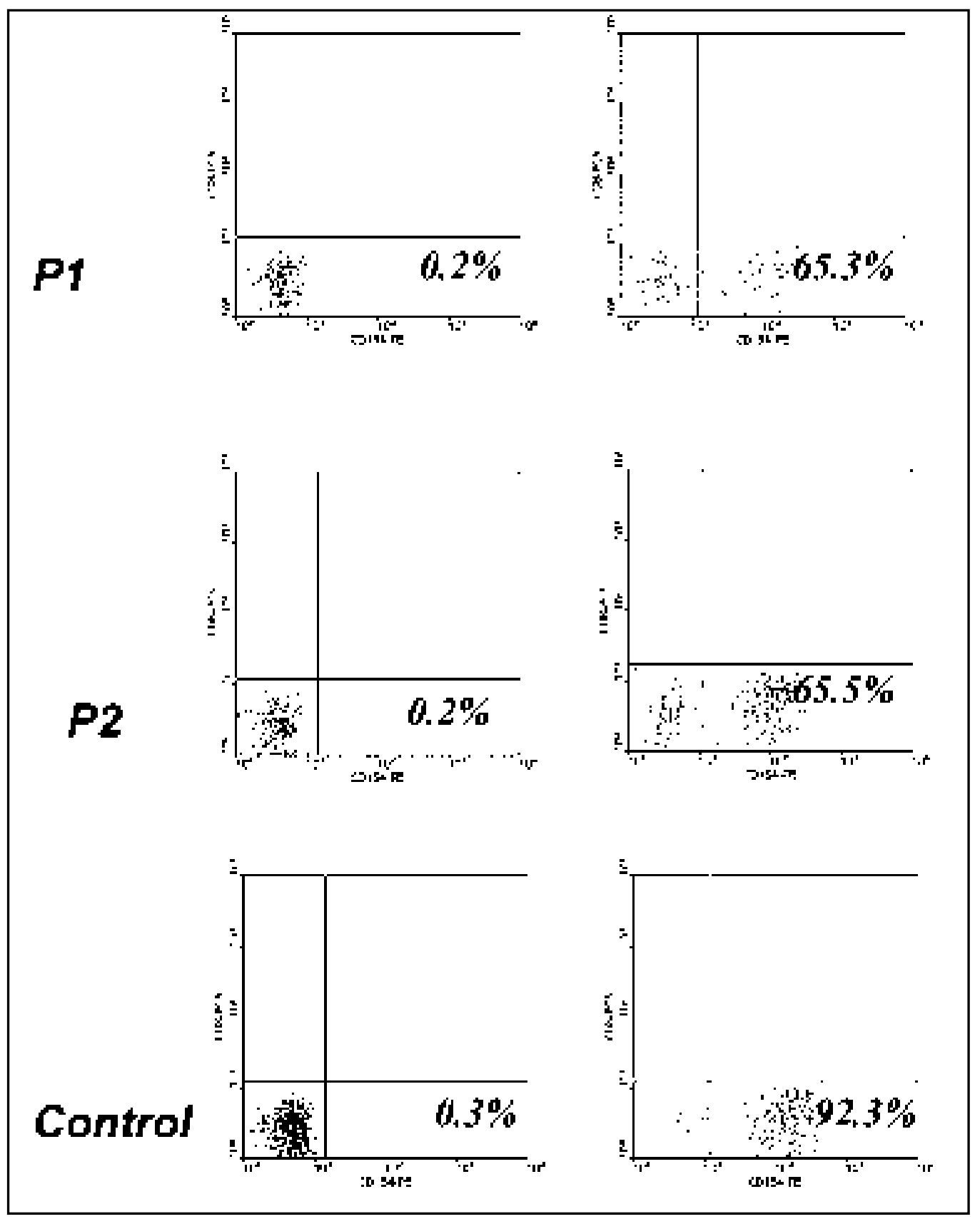

Figura 2. Análisis de la expresión de CD40L en linfocitos T CD4+ mediante citometría de flujo. La determinación se realizó en sangre total heparizinada estimulada durante $6 \mathrm{~h}$ en ausencia o presencia de PMA e Ionomicina como se describe en materiales y métodos. Para la cuantificación de la expresión de CD40L en los linfocitos T CD4+ se utilizó el siguiente panel de tres colores: CD3-FITC/CD40L-PE/CD8-TC. Esto, debido a que la estimulación durante $6 \mathrm{~h}$ con PMA e Ionomicina, induce una marcada disminución de la expresión de CD4. Los gráficos muestran el porcentaje de expresión de CD40L en los linfocitos T CD4+ de P1, P2 y el control en estado basal y tras estimulación con PMA e Ionomicina. 
SSCP y secuenciamiento del gen de la molécula CD40L La electroforesis en poliacrilamida de los productos amplificados de los 5 exones de la molécula CD40L de todos los pacientes y sus familiares sólo mostró alteraciones en el amplificado del exón 2. Se observa una banda adicional en la madre y hermana del paciente P3 de la familia 2 (Figura 3A) que no presentan ni el control ni el paciente. El secuenciamiento del ADN genómico del exón 2 del paciente P3 (Figura 3B) y su madre (Figura 3C) demostró una deleción A225 (delA225) en el paciente P3 y la calidad de portadora de la madre. El SSCP de todos los exones de la molécula CD40L de los 3 miembros de la familia 1 no mostró alteraciones.

\section{DisCUSIÓN}

Este estudio permitió hacer el diagnóstico definitivo de HIGM1 en el paciente P3 y detectar portadoras de la enfermedad en la familia 2 . No se encontraron mutaciones en los pacientes P1 y P2. Si bien el síndrome de HIGM es una enfermedad de baja frecuencia, su incidencia real no es conocida ${ }^{11}$ y el diagnóstico molecular es necesario para el manejo clínico del paciente y un adecuado consejo genético ${ }^{12,13}$. Los distintos

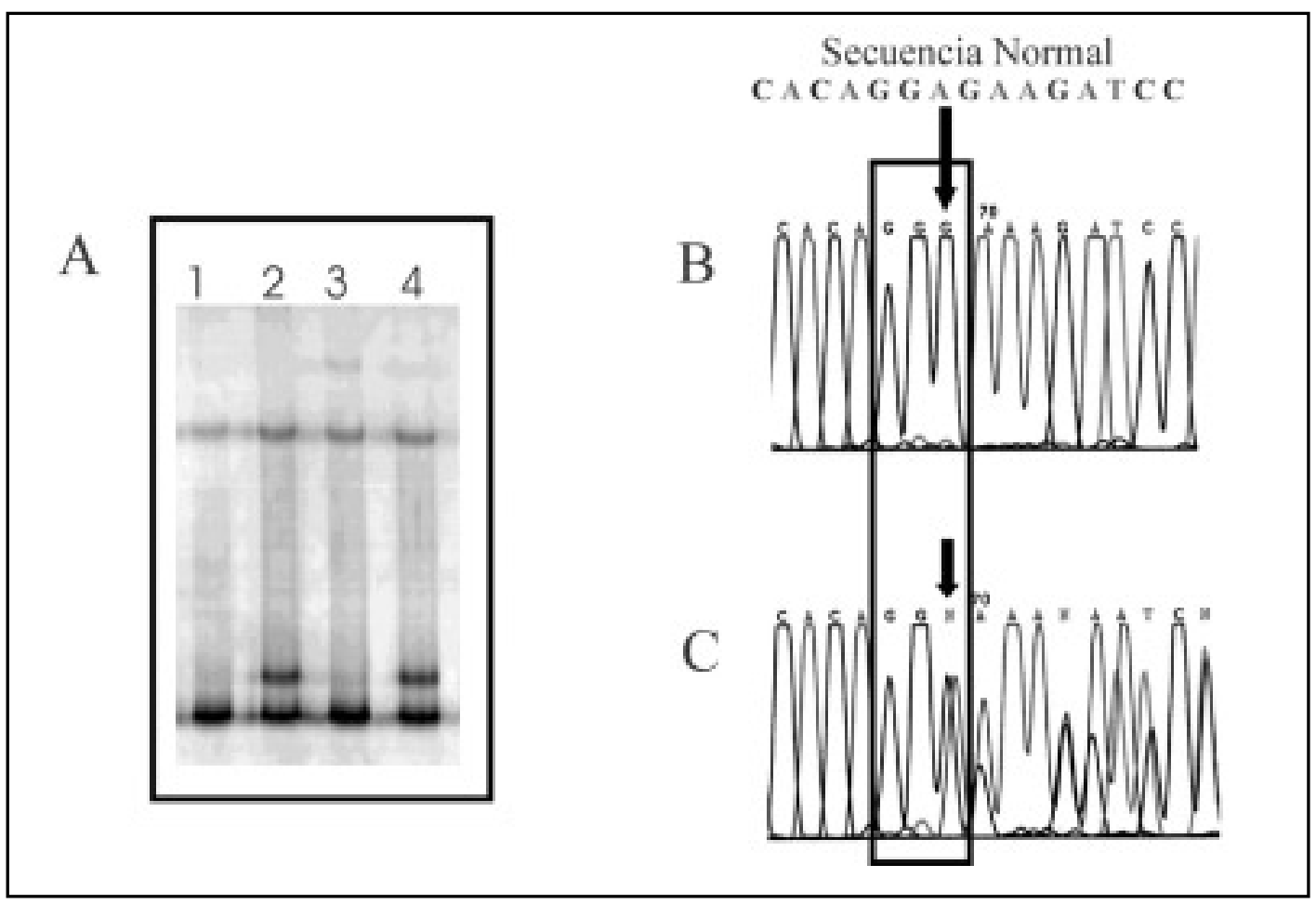

FiguRA 3. Estudios genéticos del exón 2 del gen de la molécula CD40L. (A) SSCP efectuado en condiciones no denaturantes en $6 \%$ acrilamida, 0,5 XTBE, $8 \%$ glicerol a 2 Watt, toda la noche a temperatura ambiente del exón 2 de: 1) control sano, 2) madre del paciente P3, 3) paciente P3 y 4) hermana del paciente P3. Se observa una banda adicional en la madre y hermana del paciente $\mathrm{P} 3$ que se atribuye a una cadena heterocigota formada por la secuencia normal de DNA del CD40L y una secuencia mutada. El paciente presenta bandas idénticas al control. (B) Análisis de la secuencia de parte del exón 2 del gen de la molécula CD40L en el paciente P3. La flecha muestra el sitio donde se produce la deleción de un nucleótido (delA225), en relación a la secuencia normal, que altera el marco de lectura del ADN. (C) Análisis de la misma secuencia en la madre del paciente P3 que demuestra su calidad de portadora, ya que presenta, a partir del mismo nucleótido deletado, una doble secuencia. 
tipos de HIGM tienen diferentes pronósticos, el HIGM1 y HIGM3 son inmunológica y clínicamente similares con un curso clínico más severo que HIGM2, HIGM4 e HIGM5. Levy ${ }^{14}$ describe que sólo $20 \%$ de los pacientes con HIGM ligado a X sobreviven después de los 25 años y en aquellos pacientes que se encuentran vivos a los 20 años, $75 \%$ presenta enfermedad hepática. En las series publicadas de HIGM2 2,11,15,16 el diagnóstico fue más tardío ( $2^{\mathrm{a}}$ y $3^{\mathrm{a}}$ década) y la sobrevida de los pacientes fue mayor ( $5^{\mathrm{a}}$ o $6^{\mathrm{a}}$ década) ${ }^{15}$. Desde el punto de vista terapéutico, el trasplante de médula ósea puede ser considerado en el déficit de CD40L y no cuando hay un defecto en las otras moléculas ${ }^{1}$. Se debe considerar una terapia profiláctica anti Pneumocitis carinii en pacientes con déficit de CD40L o CD40, ya que ellos tienden a hacer infecciones por este germen a pesar de la terapia de reemplazo con gamaglobulina ${ }^{14}$. Sin embargo, en los pacientes con déficit de AID no se describen infecciones por gérmenes oportunistas ${ }^{16}$.

Hasta 1996 se habían notificado al registro de IDP en Latinoamérica 34 casos de HIGM ${ }^{17}, 4$ de ellos en Chile. Actualmente, de acuerdo al Informe Anual de CHILEGID, existe un total de 8 pacientes chilenos con diagnóstico de HIGM, 2 de ellos publicados. El primero ${ }^{18}$ es un paciente de sexo femenino con expresión baja de la molécula CD40L en la superficie de los LT activados, sin defecto molecular conocido y el segundo ${ }^{19}$, un caso de HIGM1 que presenta una mutación sin sentido (nonsense) en el exón 1, que codifica para la porción transmembrana de la molécula CD40L El mayor porcentaje de mutaciones encontradas en la molécula CD40L son del tipo de cambio de sentido (missense) con 39,5\% seguidas del tipo sin sentido (nonsense) con 18,6\% y deleciones e inserciones con corrimiento del marco de lectura del ADN (deletion frameshift, insertion frameshift) con 12,8 y 10,5\%, respectivamente ${ }^{8}$. La deleción A225 encontrada en el exón 2 de la molécula CD40L del paciente P3 altera el marco de lectura del ADN (deletion frameshift) a partir del codón 75 generando 6 nuevos aminoácidos seguidos de un codón de término prematuro (Figura 4) que permiten predecir la formación de una proteína truncada que pierde parte de la región extracelular y toda la zona con homología TNF. Esta mutación es diferente a la encontrada por Rodríguez $^{19}$ y no ha sido reportada previamente en la literatura.

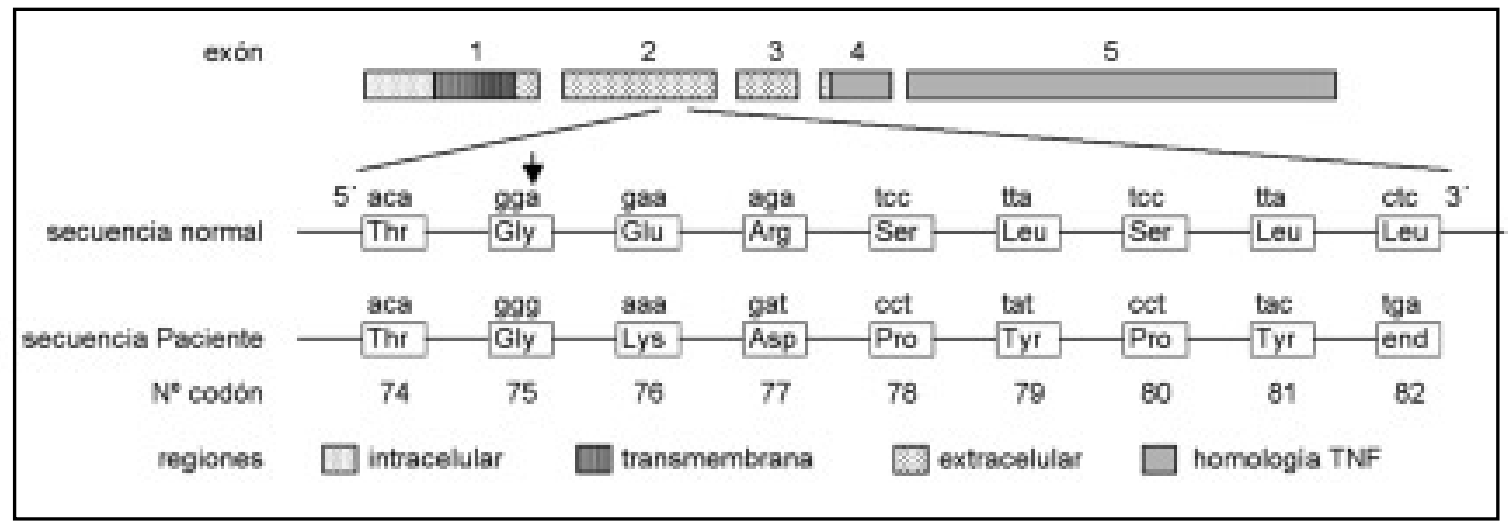

FIgURA 4. Representación esquemática del gen normal y mutado de la molécula CD40L con la secuencia de aminoácidos esperada. Se muestra una representación del gen que codifica la molécula CD40L con sus exones y regiones. La deleción del nucleótido A en la posición 225 (indicado por la flecha) altera el marco de lectura del cADN de la molécula CD40L a partir del codón 75 y produce 6 nuevos codones seguidos de un codón de término prematuro. Se predice la formación de una proteína truncada que pierde parte de la región extracelular y toda la zona con homología TNF. 
El exón 2 codifica parte de la porción extracelular única de la molécula que no está involucrada en la interacción ligando-receptor y en él se describen, al igual que en el exón 1, el 10\% del total de mutaciones ${ }^{8}$. Las bandas adicionales aparecidas en el SSCP de las portadoras se atribuyen a la movilidad electroforética del heteroduplex del amplificado del exón 2, formada por una cadena con secuencia normal y una con secuencia mutada. Debido a que fueron los amplificados de las portadoras los que entregaron los indicios de una posible mutación, queda demostrada la importancia de incluir posibles portadoras dentro del esquema de estudio molecular.

El paciente $\mathrm{P} 3$ presenta una concentración de IgA sérica normal que aumenta en los períodos de infecciones. Son muy pocos los casos que, teniendo una mutación confirmada en la molécula CD40L, presenten un fenotipo similar ${ }^{14,20}$. Se puede inducir la producción de IgA en ausencia de la interacción CD40LCD4021, sin embargo, en la gran mayoría de los pacientes con HIGM la concentración de IgA es muy baja, lo que indica que la activación de esta otra vía in vivo requiere la participación de factores adicionales.

La medición por citometría de flujo de las moléculas CD 40 y CD40L nos permite distinguir 2 posibles causas del mismo síndrome. La expresión de CD40 en el LB descarta un HIGM3 y la ausencia del CD40L en linfocitos $\mathrm{T}$ activados confirma un posible HIGM con herencia ligada al cromosoma X. En controles normales la expresión de ambos receptores oscila entre $90-97 \%$ y en la mayoría de los pacientes con HIGM por déficit de CD40L, su expresión es prácticamente nula. Generalmente, las portadoras de un HIGM ligado al

\section{REFERENCIAS}

1. Cooper M, Lanier L, Contey M, Puck J. Immunodeficiency disorders (review). Hematol 2003; 314-30.

2. Revy P, Muto T, Levy Y, Geissmann F, Piebani A, Sanal O ET al. Activation induced cytidine deaminase (AID) deficiency causes the autosomal re- cromosoma X expresan la molécula CD40L en el $50 \%$ de los LT activados ${ }^{22}$. Los dos pacientes de la familia 1 presentan una expresión parcial de la molécula CD40L, similar al descrito por otros autores en pacientes con HIGM $^{22,23}$ y pacientes con inmunodeficiencia común variable (CVID) ${ }^{24}$. Sin embargo, la probabilidad de una CVID en estos pacientes se descarta, ya que no cumplen con los criterios diagnósticos. La expresión parcial de la molécula CD40L en los linfocitos T activados no descarta el diagnóstico de HIGM1, ya que pacientes con defectos genéticos conocidos en la molécula CD40L pueden expresar una molécula mutante capaz de ser reconocida por anticuerpos específicos ${ }^{22,23,25}$. También se ha descrito que mutaciones en la zona de los intrones del ADN genómico de la molécula CD40L puede generar, por splicing alternativo, heterotrímeros parcialmente expresados pero no funcionales ${ }^{25,26}$. Los estudios genéticos por SSCP de toda la molécula CD40L en la familia 1 dio resultados normales. Teniendo en cuenta la expresión de la molécula por citometría de flujo y dado que la sensibilidad de la técnica SSCP es de aproximadamente $75 \% 27$, no podemos descartar un defecto en el gen del CD40L. Sin embargo, considerando estos resultados, la consanguinidad de los padres y el curso clínico más benigno de los pacientes P1 y P2 podemos postular un HIGM con una forma de herencia autosómica recesiva tipo HIGM2, HIGM4 o HIGM5.

En conclusión, este estudio permitió hacer el diagnóstico definitivo HIGM1 en el paciente P3 y confirmar que su madre y hermana son portadoras de la misma deleción. Es necesario efectuar otros estudios en los pacientes P1 y P2 que permitan demostrar la causa molecular de su síndrome.

cessive form of the hyper-IgM syndrome (HIGM2). Cell 2000; 102: 565-75.

3. Ferrari S, Giluani S, Insalaco A, Al-Ghonaium A, Soresina AR, LOUBSER M ET AL. Mutations of CD40 gene cause an autosomal recessive form of immunodeficiency with hyper IgM. Proc Natl Acad Sci 2001; 98: 12614-9. 
4. Imai K, Catalán N, Piebani A, Marodi L, Sanal O, KUMAKI S ET AL. Hyper-IgM syndrome type 4 with a B lymphocyte-intrinsic selective deficiency in Ig class-switch recombination. J Clin Invest 2003; 112: 136-42.

5. Imai K, Slupphaug G, Lee W, Revy P, Nonoyama $\mathrm{S}$, Catalán N ET al. Human uracil-DNA glycosylase deficiency associated with profoundly impaired immunoglobulin class-switch recombination. Nature Immunol 2003; 10: 1023-8.

6. Jain A, Ma C, Lui S, Brown M, Cohen J, Strober W. Specific missense mutations in NEMO result in hyper-IgM syndrome with hypohydrotic ectodermal dysplasia. Nature Immunol 2001; 2: 223-8.

7. Gulino AV, Notarangelo LD. Hyper IgM syndromes (review). Curr Opin Rheumatol 2003; 15: 422-9.

8. Notarangelo L, Peitsch M, Abrahamsen T, Bachelot C, Bordigoni P, CANT A et al. CD40Lbase: a database of CD40L gene mutations causing $\mathrm{X}$ linked hyper IgM syndrome. Immunol Today 1996; 17: 511-6.

9. Conley ME, Notarangelo LD, Etzioni A. Diagnostic criteria for Primary Immunodeficiencies. Clin Immunol 1999; 93: 190-7.

10. O'Gorman M, Zaas D, Paniagua M, Corrochano V, Scholl P, Pachman L. Development of a rapid whole blood flow cytometry procedure for the diagnosis of X-linked hyper-IgM syndrome patients and carriers. Clin Immunol 1997; 5: $172-81$.

11. Minegishi Y, Lavoie A, Cunningham-Rundies C, BeDARD PM, HÉBert J, Cote L et al. Mutations in activation-induced cytidine deaminase in patients with hyper IgM syndrome. Clin Immunol 2000; 97: 203-10.

12. Burke W. Genetic Testing. N Engl J Med 2002; 347: 1867-75.

13. WeIL J. Genetic counselling in the era of genomic medicine: As we move towards personalized medicine, it becomes more important to help patients understand genetic tests and make complex decisions about their health. EMBO Rep 2002; 3: 590-3.

14. Levy J, Espanol-Boren T, Thomas C, Fischer A, Pierangelo T, Bordigoni P et al. Clinical spectrum of X-linked hyper-IgM syndrome. J Pediatr 1997; 131: 47-54.
15. Zhu Y, Nonoyama S, Morio T, Muramatsu M, Honjo T, Mizutani S. Type two hyper-IgM syndrome caused by mutation in activationinduced cytidine deaminase. J Med Dent Sci 2003; 50: 41-6.

16. Quartier P, Bustamante J, Sanal O, Piebani A, Debre M, Devile A et al. Clinical, immunologic and genetic analysis of 29 patients with autosomal recessive hyper-IgM syndrome due to ActivationInduced Cytidine Deaminase deficiency. Clin Immunol 2004; 110: 22-9.

17. Zelazko M, Carneiro-Sampaio M, Cornejo De Luigi M, García De Olarte D, Porras Madrigal O, Berrón PÉreZ R ET AL. Primary Immunodefiency Diseases in Latin America: First Report from eight countries participating in the LAGID. Clin Immunol 1998; 18: 161-6.

18. Quezada A, Norambuena $X$, González R, Ramos $S$ Chávez A, González S. Non-X-linked hyperimmunoglobulin $\mathrm{M}$ syndrome with chronic interstitial pneumonitis. Invest Allergol Clin Immunol 2000; 10: $375-9$.

19. Rodríguez C, Carrión F, Marinovic Ma, Chávez E, Preisler J, Pooley F et al. X-linked hyper IgM syndrome associated to sclerosing cholangitis and gallbladder cancer. Rev Méd Chile 2003; 131: 303-8.

20. Atkinson P, Smith C, Hsu Y, Garber E, Su L, HowARD T ET AL. Leucocyte transfusion-associated granulocyte responses in a patient with X-linked hyper-IgM syndrome. J Clin Immunol 1998; 18: 430-9.

21. Litinsky M, Nardem B, Hilbert D, He B, Schaffer A, CASALI P ET AL. DCs induce CD40-independent immunoglobulin class switching through BLyS and APRIL. Nature Immunol 2002; 3: 822-9.

22. Callard R, Smith S, Herbert J, Morgan G, PadayaChee M, Lederman S et al. CD40 ligand (CD40L) expression and $\mathrm{B}$ cell function in agammaglobulinemia with normal or elevated levels of IgM (HIG). Comparison of X-linked, Autosomal Recessive, and Non-X-linked forms of disease and obligate carriers. J Immunol 1994; 153: 3295-306.

23. Seyama K, Nonoyama S, Gangsaas I, Holemnaugh D, Pabst H, ARUFFo A et al. Mutations of the CD40 ligand gene and its effect on CD40 ligand expression in patients with X-linked hyper IgM syndrome. Blood 1998; 92: 2421-34. 
24. Farrigton M, Grosmaire L, Nonoyama S, Fischer S, Howenbaugh D, Ledbetter J et al. CD40 ligand expression is defective in a subset of patient with common variable immunodeficiency. Proc Nat Acad Sci USA 1994; 91: 1099-103.

25. Zhu X, Chung I, O’Gorman MR, Schol PR. Coexpression of normal and mutated CD40 ligand with deletion of a putative RNA lariat branchpoint sequence in X-linked hyper-IgM syndrome. Clin Immunol 2001; 99: 334-9.
26. Seyama K, Osborne W, Ochs H. CD40 ligand responsible for $\mathrm{X}$-linked hyper-IgM syndrome associate with wild type CD40 ligand. J Biol Chem 1999; 274: 11310-320.

27. Puck J, Pepper A, Henthorn P, Candotti F, Isakov J, WhITMAN T ET AL. Mutation analysis of IL2RG in human X linked severe combined immunodeficiency. Blood 1997; 89: 1968-77. 